\title{
A new method to characterize target location in lung cancer patients treated with stereotactic body radiation therapy
}

\author{
Martina Descovich • Sebastian A. Dionisio • \\ Tina Dasgupta $\cdot$ Sue S. Yom
}

Received: 7 December 2011 / Accepted: 25 December 2011 / Published online: 14 February 2012

(C) Springer-Verlag 2012

\begin{abstract}
Purpose: Stereotactic body radiation therapy is an emerging technique in the treatment of peripheral lung tumors. However, due to early reports of increased toxicity, the treatment of central lung lesions with hypo-fractionated high-dose regimen remains controversial. Thus far, lung lesions have been defined as either central or peripheral depending solely on their nearest distance from the major airways. The goal of this study is to develop a quantitative method to categorize the location of lung lesions mapped in three-dimensional space. Furthermore, a set of parameters are proposed for assessing risk factors based on target locations.

Methods and Materials: A MATLAB program was developed to quantify the distance between the tumor and the airways, and to calculate the percentage of the target volume lying within a given distance from the main bronchial tree. The program was tested on 20 patients with centrally located lung lesions treated with CyberKnife. A dosimetric analysis was performed to investigate the relationship between dose delivered to the critical structures and target location.

Results: The absolute target volume located within a distance of $20 \mathrm{~mm}$ from the airways was used to quantify the tumor proximity to the bronchial tree. A strong correlation was found
\end{abstract} \footnotetext{
Department of Radiation Oncology, University of California San Francisco, 1600 Divisadero, Suite H1031, San Francisco, CA 94143-1708, USA e-mail: mdescovich@radonc.ucsf.edu

\section{S. A. Dionisio}

Neuroscience Imaging Center, University of California San Francisco, San Francisco, CA, USA
}

between this parameter and the dose delivered to the critical organs, showing that a detailed knowledge of target location can be used to assess the risk of toxicity during the planning stage. Conclusions: We have developed a tool to quantify the lesion proximity to the bronchial tree. We expect that riskadapted strategies, accounting for the exact geometric relationship between the tumor and the airways, may improve the management of centrally located lung lesions.

Keywords Lung · Central lesion · Stereotactic body radiotherapy $(\mathrm{SBRT}) \cdot$ CyberKnife

\section{Introduction}

In recent years, stereotactic body radiation therapy (SBRT) has emerged as a new treatment modality in the management of patients with primary and metastatic lung cancer. Although surgical resection remains the standard of care for early-stage lung cancer patients, clinical studies on medically inoperable patients treated with SBRT are showing very promising results [1-3]. In particular, results from the first North American multicenter prospective study demonstrated a 3-year primary tumor control rate of $97.6 \%$, a 3-year local control rate of $90.6 \%$, and an overall survival rate at 3 years of $55.8 \%$ for patients with early-stage peripheral lesions [4]. In conventional radiation therapy series, local failure rates of $42 \%$ and 5 -year survival rates of $21-32 \%$ have been observed [5-8]. The favorable outcomes of SBRT are due to the high biologic-equivalent dose regimens and the short treatment package times (one to five fractions) made possible by this modality. For example, in the Radiation Therapy Oncology Group (RTOG) 0236 trial, a dose regimen of $60 \mathrm{~Gy}$ in three fractions was employed. 
Today, thoracic SBRT treatments can be delivered using a variety of treatment planning platforms and radiation delivery units [9-11]. All modalities share the capability of delivering radiation via numerous non-coplanar/non-isocentric small beams, and monitoring the target position with high accuracy just prior to radiation delivery. Among these, the CyberKnife system (Accuray Inc., Sunnyvale, California) offers a fully integrated technology for synchronizing respiratory-induced target motion with radiation delivery [3]. The combination of multiple highly focused beams and motion tracking enables reducing the volume of normal tissue exposed to high doses of radiation, thus allowing safe delivery of these high-dose regimens. An example of dose distribution for a patient treated with CyberKnife SBRT (50 Gy in five fractions) is presented in Fig. 1. A steep dose fall-off in the direction of the airways is achieved. The 25 Gy isodose line (i.e., $50 \%$ of the prescription dose) is touching the airways at a distance of $5 \mathrm{~mm}$ from the planning target volume (PTV).

The treatment of patients who present a lesion in the central portion of the lung (hilar or pericentral) by means of SBRT remains controversial. In 2006, Timmerman et al. reported excessive toxicity when treating central tumors with an SBRT protocol of $60 \mathrm{~Gy}$ in three fractions [12]. The authors reported that the lesion location within the chest (central vs. peripheral) was a statistically significant factor in predicting toxicity, and recommended that patients with centrally located lesions should be excluded from standard SBRT protocols.

According to the guidelines of the current generation of lung SBRT protocols from the RTOG, perihilar/central lesions are those located within $20 \mathrm{~mm}$ of the carina or main bronchus. Figure 2 shows a schematic diagram of the perihilar/central lung region, and depicts the zone of the proximal bronchial tree. A current RTOG protocol (RTOG 0813)

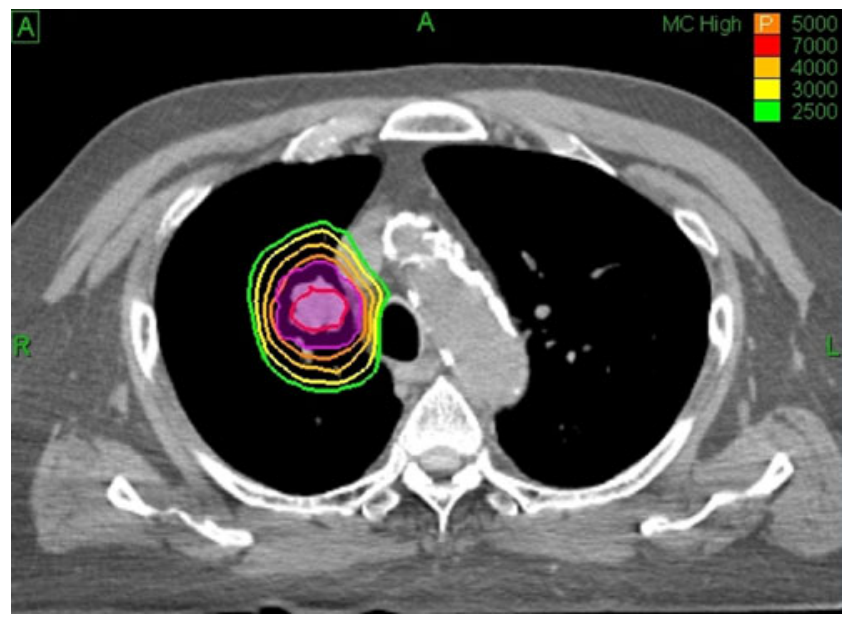

Fig. 1 Example of dose distribution for a patient with a central lung lesion treated with CyberKnife SBRT

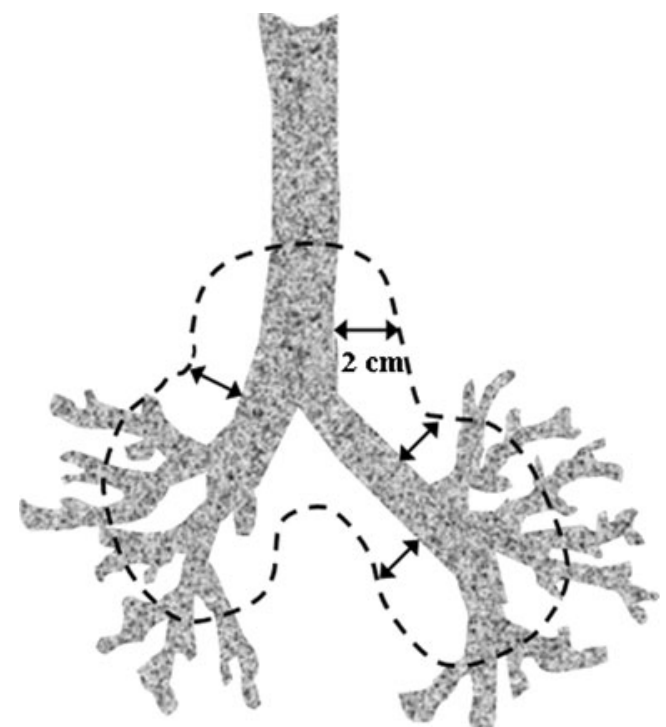

Fig. 2 Schematic illustration of the bronchial tree. The dashed line represents the zone of the proximal bronchial tree according to the definition in reference [12]

is utilizing a dose escalation scheme to characterize the toxicities when treating in this area and to determine what a safe dose might be. Off protocol, lesions within this zone are typically prohibited from treatment with SBRT to avoid the occurrence of high grade toxicity.

In routine clinical practice, the tumor location is defined as central if any portion of the tumor margin is at the boundary of the proximal bronchial tree zone. According to this definition, both tumors encroaching upon the central lung region and tumors fully enclosed within it are considered to fall into a similar risk category. Some authors have proposed more precise classification schemes. For example, Song et al. subdivided central tumors into peribronchial and endobronchial tumors, depending whether the lesion was within the zone of the proximal bronchial tree or immediately adjacent (and invading) the main bronchus [13]. De La Fuente Herman et al. classified tumor location by dividing the chest cavity into three equal portions (peripheral right, central, and peripheral left) and considered a tumor as central if its margin touched the central region [14]. Although more descriptive, these methods do not provide a quantitative way to accurately characterize lesion location.

In order to study the occurrence of toxicity as a function of target location and to enable the development of predictive toxicity models, we believe a more rigorous approach is needed. The main goal of this study was to establish a quantitative definition of centrality based on a lesion's exact position relative to the bronchial tree. The developed method was applied to a clinical database of patients treated with CyberKnife lung SBRT. The relationship between target 
location and dosimetric parameters was evaluated using this quantitative definition.

\section{Methods and materials}

\section{Program description}

To characterize the target location in relationship to the bronchial tree, a MATLAB program was developed. MAT$\mathrm{LAB}$ was chosen as the coding language because of its ease in dealing with large arrays and its built-in functions for managing DICOM files. Most aspects of the treatment plan, including CT images and segmented anatomical structures, can be exported from the treatment planning system to a DICOM file and accessed through MATLAB. In particular, the radiation therapy segmented structures (RTSS) file exported from the CyberKnife planning platform (Multiplan, version 3.5) contains the $x, y, z$ coordinates of the boundary of all segmented structures.

Separate functions were written to calculate: (1) the minimum and maximum distances between two structures, (2) the global distance between the centers of two structures, (3) the distance in the superior-inferior direction between the centers of two structures, and (4) the percent of a structure's volume within a certain distance (overlap) from a second structure. To facilitate data handling, the process of importing the DICOM files and collecting coordinates for selected structures was automated. Since all coordinates on a given CT slices have the same $z$ value, the program starts by sorting coordinates by CT slice. A regular mesh of userdefined size is created for each CT slice that spans from the minimum to the maximum $x$ and $y$ values. All points in the mesh are then tested with a built-in MATLAB function (inpolygon) which determines if points are located within the polygon defined by the vertices of the structure. All points that are contained within the structure are stored in an array along with all points on the boundary of the contour. The minimum distances between these points and those on the boundary of the second structure are calculated. The numbers of points with a minimum distance lower than the user-defined distance are tallied. The percentage of volume within a certain distance of the second structure is then defined as the number of tallied points divided by the total number of points within the first structure.

Functions were tested for accuracy by importing userdrawn structures where critical values were known beforehand. Structures of simple geometry such as circles and squares were created in MultiPlan with known minimum and maximum distances and then verified with the MATLAB functions. To verify the overlap function, several simple shapes were drawn where a quarter of the volume and half the volume were set at predetermined distances from a second structure. Distances between structures were also determined using the ruler function in Multiplan.

\section{Patient data}

The program was tested on 20 patients treated for metastatic or primary lung lesions in the central and hilar portion of the lung. All patients received CyberKnife SBRT with different dose and fractionation schemes, ranging from one to five fractions (Table 1). For each dose regimen, the biologically equivalent dose (BED) corresponding to $2 \mathrm{~Gy} /$ fraction was calculated using the formula: $\mathrm{BED}=\mathrm{nd}(1+\mathrm{d} /(\alpha / \beta))$, where $\mathrm{n}=$ number of fractions; $\mathrm{d}=$ dose per fraction; and $\alpha / \beta=10$ for acute reacting tissue. At the time of treatment, all of these lesions were categorized as central (per the RTOG definition) by the treating physician, based on a visual estimation of the distance from the bronchial tree. The distances between the lesion and the airways were measured using the ruler function in Multiplan. For 18 patients, the hand-calculated distances $\left(d_{\text {hand }}\right)$ agreed to those calculated by MATLAB to less than $0.5 \mathrm{~mm}$. For two patients, the hand-calculated distances were 2 and $3 \mathrm{~mm}$ larger than those calculated by the program, showing a certain uncertainty associated with the manual method. Except for one patient, the minimum distance between the target and the airways was less than $1 \mathrm{~mm}$ for all considered cases.

For each patient, the PTV, the airway, and the carina were contoured in the CyberKnife treatment planning platform. To standardize the results, the carina was defined as three slices above and three slices below the tracheal bifurcation (the slice thickness in the CT scan was $1.5 \mathrm{~mm}$ ). The structure of the major airway was defined as the inner section of the main bronchus up to the second branch nearest the lesion. Other contoured structures included: esophagus, lungs, spinal cord, heart, and respiratory tract. For each patient, the minimum distance $\left(d_{\min }\right)$ between the PTV and the airways was calculated, as well as the fraction of PTV volume within a distance of $5 \mathrm{~mm}\left(f_{5}\right), 10 \mathrm{~mm}\left(f_{10}\right)$, and $20 \mathrm{~mm}\left(f_{20}\right)$ from the airways. Other parameters calculated by the MATLAB program were the distance between the center of the PTV and the center of the carina $\left(d_{\text {car }}\right)$, and the distance between the center of the PTV and the center of the carina in the superior-inferior direction $\left(d_{\mathrm{car}-\mathrm{S} / \mathrm{I}}\right)$.

The absolute volume of PTV in units of cubic centimeter (cc) within a distance of 5, 10, and $20 \mathrm{~mm}$ from the airways was calculated by multiplying the PTV volume by $f_{5}, f_{10}$, and $f_{20}$, respectively. Two parameters were used to categorize the patients: (1) the fractional PTV volume within $20 \mathrm{~mm}$ from the airways and (2) the absolute PTV volume within $20 \mathrm{~mm}$ from the airways.

For each plan, several dosimetric parameters were recorded and compared, including target dose, target coverage (percentage of PTV volume receiving the total 
Table 1 Lesions characteristics and treatment parameters

\begin{tabular}{|c|c|c|c|c|c|c|c|c|c|c|c|}
\hline Patient no. & PTV vol. (cc) & Total dose (Gy) & $\mathrm{Fx}$ & BED (Gy) & $d_{\min }(\mathrm{mm})$ & $d_{\text {hand }}(\mathrm{mm})$ & $d_{\text {car }}(\mathrm{mm})$ & $d_{\text {car-S/I }}(\mathrm{mm})$ & $f_{5}$ & $f_{10}$ & $f_{20}$ \\
\hline 1 & 3.4 & 20 & 2 & 40 & 19.0 & 19.5 & 59.0 & 17.6 & 0.00 & 0.00 & 0.02 \\
\hline 2 & 492.0 & 30 & 5 & 48 & 0.0 & 0 & 71.0 & 9.5 & 0.02 & 0.06 & 0.16 \\
\hline 3 & 440.6 & 10 & 2 & 15 & 0.3 & 0 & 57.6 & 31.8 & 0.04 & 0.09 & 0.21 \\
\hline 4 & 173.7 & 40 & 5 & 72 & 0.2 & 0 & 79.0 & 33.3 & 0.02 & 0.07 & 0.22 \\
\hline 5 & 205.1 & 20 & 5 & 28 & 0.4 & 0 & 60.9 & 15.8 & 0.05 & 0.10 & 0.24 \\
\hline 6 & 72.2 & 50 & 5 & 100 & 0.4 & 3.5 & 69.9 & 49.6 & 0.03 & 0.10 & 0.33 \\
\hline 7 & 47.7 & 30 & 3 & 60 & 0.5 & 0 & 56.5 & 15.8 & 0.05 & 0.12 & 0.36 \\
\hline 8 & 61.2 & 24 & 2 & 52.8 & 0.0 & 0 & 90.2 & 35.1 & 0.07 & 0.19 & 0.52 \\
\hline 9 & 124.0 & 30 & 5 & 48 & 0.2 & 0 & 56.5 & 45.3 & 0.10 & 0.23 & 0.53 \\
\hline 10 & 44.1 & 60 & 5 & 132 & 0.0 & 0 & 50.6 & 42.8 & 0.09 & 0.21 & 0.56 \\
\hline 11 & 17.4 & 50 & 5 & 100 & 0.1 & 0 & 77.2 & 49.2 & 0.09 & 0.26 & 0.70 \\
\hline 12 & 63.4 & 50 & 5 & 100 & 0.0 & 0 & 40.8 & 9.2 & 0.13 & 0.33 & 0.77 \\
\hline 13 & 21.3 & 20 & 1 & 60 & 0.1 & 1.8 & 70.4 & 43.7 & 0.13 & 0.32 & 0.78 \\
\hline 14 & 11.7 & 20 & 1 & 60 & 0.2 & 0 & 52.7 & 38.6 & 0.17 & 0.40 & 0.93 \\
\hline 15 & 32.2 & 50 & 5 & 100 & 0.0 & 0 & 62.7 & 45.3 & 0.38 & 0.64 & 0.97 \\
\hline 16 & 10.6 & 16 & 1 & 41.6 & 0.0 & 0 & 22.1 & 4.6 & 0.23 & 0.57 & 1.00 \\
\hline 17 & 43.2 & 30 & 3 & 60 & 0.4 & 0 & 21.9 & 20.9 & 0.30 & 0.90 & 1.00 \\
\hline 18 & 84.4 & 20 & 2 & 40 & 0.4 & 0 & 23.1 & 21.6 & 0.29 & 0.89 & 1.00 \\
\hline 19 & 14.3 & 50 & 5 & 100 & 0.2 & 0 & 45.4 & 37.3 & 0.44 & 0.75 & 1.00 \\
\hline 20 & 5.9 & 48 & 4 & 105.6 & 0.1 & 0 & 29.8 & 7.9 & 0.44 & 0.86 & 1.00 \\
\hline
\end{tabular}

Fx number of fractions, BED biologically equivalent dose $d_{\min }$ minimum distance between the PTV and the airways, $d_{\text {hand }}$ minimum distance between the PTV and the airways determined by hand using the ruler function in Multiplan, $d_{c a r}$ distance between the center of the PTV and the center of the carina, $d_{c a r-S / I}$ distance between the center of the PTV and the center of the carina in the superior-inferior direction, $f_{5}$ fraction of PTV volume within a distance of $5 \mathrm{~mm}$ from the airways, $f_{10}$ fraction of PTV volume within a distance of $10 \mathrm{~mm}$ from the airways, $f_{20}$ fraction of PTV volume within a distance of $20 \mathrm{~mm}$ from the airways

prescription dose) and dose to the critical structures. The following parameters were used to evaluate the dose delivered to the critical structures:

1. $\mathrm{D} 1_{\mathrm{cc}}$ is the largest dose received by $1 \mathrm{cc}$ of a critical structure;

2. D $10 \%$ is the largest dose received by $10 \%$ of the volume of a critical structure.

To account for differences in dose schemes, the parameters $\mathrm{D} 1_{\mathrm{cc}}$ and $\mathrm{D} 10_{\%}$ were expressed relative to the total prescription dose. Based on the dose level observed in the critical structures, a risk level was associated to each group.

\section{Results}

Fractional volume of PTV within the zone of the proximal bronchial tree

Table 1 reports information on the selected patient population including PTV volume, total prescribed dose, number of fractions, BED, $d_{\text {min }}, d_{\text {hand }}, d_{\text {car }}, d_{\text {car-S/I }}, f_{5}, f_{10}$, and $f_{20}$. Patients are sorted in order of ascending $f_{20}$. From these data, it appears that our physicians tended to authorize treatment for smaller lesions fully inside the proximity zone and larger lesions which lay only partially inside the proximity zone. In addition, the variations in dose scheme were more related to target dimensions, rather than proximity to the bronchial tree. No significant difference in the dose to the critical structures was observed by analyzing the data as a function of the fractional volume of PTV within the proximity zone. This indicates that treating physicians based their prescriptions on what could be achieved respecting adjacent normal organ tolerance.

Absolute volume of PTV within the zone of the proximal bronchial tree

In order to evaluate risk factors for patients with central lung lesions, we considered the absolute volume of target within a distance of $20 \mathrm{~mm}$ from the airways. Based on this criterion, patients were divided in four groups. A risk level was associated to each group:

1. Risk level 1: volume of PTV inside the $20-\mathrm{mm}$ proximity zone $<10 \mathrm{cc}$;

2. Risk level 2: volume of PTV inside the 20-mm proximity zone $\geq 10 \mathrm{cc}$ and $<20 \mathrm{cc}$; 
Table 2 Dosimetric parameters used to evaluate the dose to the critical structures

Risk group Patient number $\quad \mathrm{V}_{20}(\mathrm{cc}) \quad$ Esophagus D1 $1_{\mathrm{cc}}(\%) \quad$ Cord D1 $1_{\mathrm{cc}}(\%) \quad$ Resp. tract D1 $1_{\mathrm{cc}}(\%) \quad H e a r t \mathrm{D} 1_{\mathrm{cc}}(\%) \quad$ Lung D10\% $(\%)$

\begin{tabular}{|c|c|c|c|c|c|c|c|}
\hline \multirow[t]{2}{*}{ Level 1} & 1 & 0.1 & 2.0 & 9.0 & 1.8 & 13.8 & 18.9 \\
\hline & 20 & 5.9 & 13.2 & 16.4 & 51.3 & 18.0 & 18.6 \\
\hline \multirow[t]{6}{*}{ Level 2} & 16 & 10.6 & 15.5 & 15.0 & 82.5 & 13.6 & 15.7 \\
\hline & 14 & 10.9 & 1.9 & 15.0 & 27.8 & 30.5 & 22.6 \\
\hline & 11 & 12.3 & 5.1 & 24.7 & 8.4 & 7.5 & 5.2 \\
\hline & 19 & 14.3 & 22.0 & 15.7 & 2.2 & 15.9 & 12.0 \\
\hline & 13 & 16.7 & 2.5 & 10.9 & 43.8 & 60.5 & 38.2 \\
\hline & 7 & 17.3 & 26.5 & 13.7 & 73.6 & 23.7 & 35.5 \\
\hline \multirow[t]{6}{*}{ Level 3} & 6 & 24.1 & 29.0 & 16.4 & 74.2 & 82.2 & 32.3 \\
\hline & 10 & 24.7 & 27.1 & 35.2 & 20.6 & 16.8 & 16.4 \\
\hline & 15 & 31.1 & 51.7 & 20.2 & 39.4 & 75.3 & 38.4 \\
\hline & 8 & 31.6 & 19.7 & 12.5 & 91.7 & 48.5 & 53.9 \\
\hline & 4 & 38.8 & 40.9 & 41.8 & 78.8 & 69.7 & 69.9 \\
\hline & 17 & 43.2 & 52.4 & 16.7 & 133.3 & 16.7 & 21.3 \\
\hline \multirow[t]{6}{*}{ Level 4} & 12 & 48.9 & 12.3 & 17.8 & 84.9 & 9.2 & 38.0 \\
\hline & 5 & 49.1 & 47.1 & 16.2 & 69.1 & 85.3 & 10.8 \\
\hline & 9 & 66.1 & 64.5 & 42.5 & 126.2 & 61.3 & 64.9 \\
\hline & 2 & 77.7 & 66.7 & 37.8 & 81.1 & 88.9 & 91.1 \\
\hline & 18 & 84.4 & 88.7 & 20.0 & 150.9 & 18.0 & 30.8 \\
\hline & 3 & 92.2 & 91.1 & 37.5 & 100.0 & 56.2 & 84.3 \\
\hline
\end{tabular}

$V_{20}$ absolute volume of target inside the 20-mm proximity zone, $D 1_{c c}$ largest dose delivered to $1 \mathrm{cc}$ of a given critical structure, $D 10_{\%}$ largest dose delivered to $10 \%$ of a given critical structure, Resp. Tract respiratory tract

3. Risk level 3: volume of PTV inside the 20-mm proximity zone $\geq 20 \mathrm{cc}$ and $<45 \mathrm{cc}$;

4. Risk level 4: volume of PTV inside the 20-mm proximity zone $\geq 45 \mathrm{cc}$.

Each group consisted of six patients, except for group 1 which had only two patients. Therefore, a statistical analysis

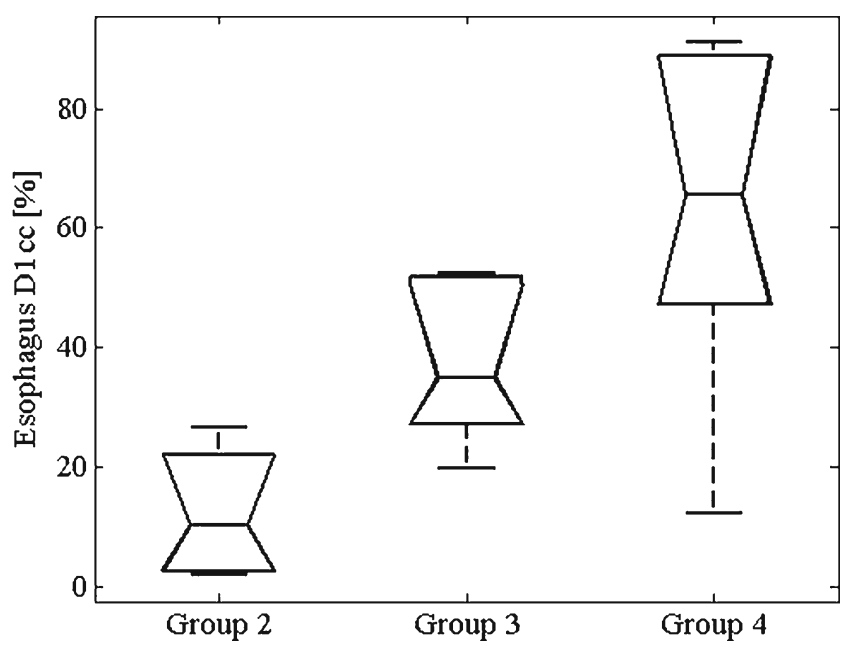

Fig. 3 Box plot analysis for dose to $1 \mathrm{cc}\left(\mathrm{D} 1_{\mathrm{cc}}\right)$ of esophagus as a function of the risk group ( $p$ value $=0.009$ ). $\mathrm{D} 1_{\mathrm{cc}}$ is expressed as a percentage of the total dose was performed for groups 2, 3, and 4. The Kruskal-Wallis test was used to determine whether changes in dose to the critical structures were significantly different for the risk level groups. The Kruskal-Wallis is a non-parametric method to test the hypothesis that three samples originate from the same population. The test is positive if $p$ value $<0.05$ is obtained.

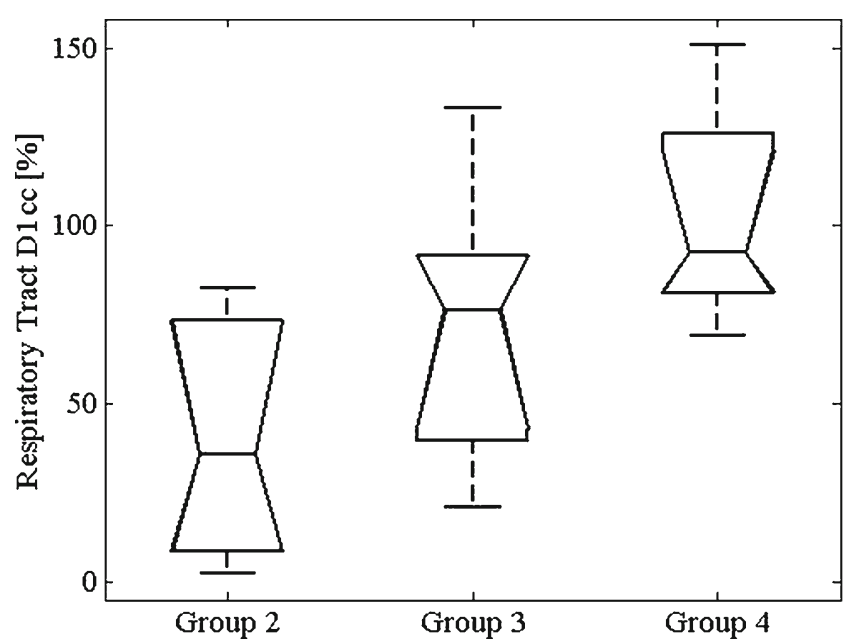

Fig. 4 Box plot analysis for dose to $1 \mathrm{cc}\left(\mathrm{D} 1_{\mathrm{cc}}\right)$ of respiratory tract as a function of the risk group ( $p$ value $=0.039) . \mathrm{D} 1_{\mathrm{cc}}$ is expressed as a percentage of the total dose 


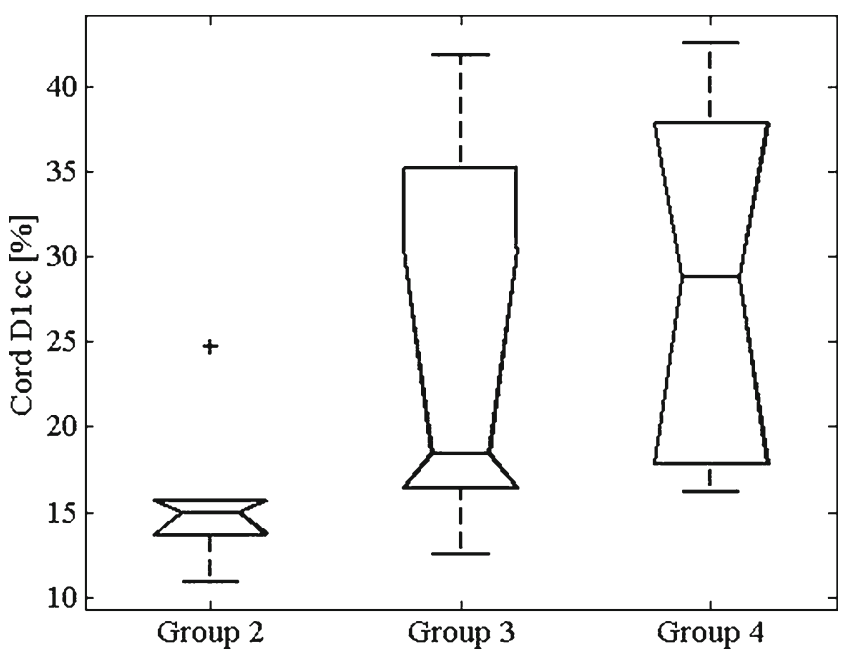

Fig. 5 Box plot analysis for dose to $1 \mathrm{cc}\left(\mathrm{D} 1_{\mathrm{cc}}\right)$ of spinal cord as a function of the risk group ( $p$ value $=0.046)$. D $1_{c c}$ is expressed as a percentage of the total dose

Patients in the low-risk groups had smaller PTV volume, and received higher dose per fraction, compared with the higher-risk groups. The average target volume was 21, 71, and $235 \mathrm{cc}$ ( $p$ value $=0.002)$ in groups 2,3 , and 4, respectively. The average dose per fraction was 14,10 , and 7 Gy ( $p$ value $=0.014$ ) in groups 2,3 , and 4 , respectively. For all targets, a PTV coverage $>94 \%$ was achieved, with no significant differences among the different groups. The average PTV coverage was 97\%, 98\%, and 97\% in group 2, 3, and 4, respectively.

Table 2 reports the results for the dose to the critical structures (spinal cord, esophagus, respiratory tract, heart, and ispilateral lung) sorted and grouped as a function of ascending absolute PTV volume within the $20-\mathrm{mm}$ proximity zone. The parameters $\mathrm{D} 1_{\mathrm{cc}}$ and $\mathrm{D} 10_{\%}$ are reported as percentage of the total prescription dose. In this way, dose fall off away from the target was evaluated independently of the prescription dose.

Statistically significant differences in the dose to the critical structures emerged among the different risks groups. Figures 3, 4 , and 5 show a "box-plot" analysis for $\mathrm{D} 1_{\mathrm{cc}}$ of the esophagus, respiratory tract, and spinal cord, respectively. In these plots, the box has lines at the 25 th percentile, 50 th percentiles (median), and 75 th percentile values. For all structures, $\mathrm{D} 1_{\mathrm{cc}}$ increases significantly depending on the risk level. Table 3 reports the average values, standard deviation, and statistical significance (Kruskal-Wallis $p$ value) for the dose to esophagus, cord, respiratory tract, heart, and ipsilateral lung.

\section{Discussion}

Recent studies have demonstrated that the lesion location (central versus peripheral) and the target volume size are strong predictors of toxicity when treating lung tumors with a hypo-fractionated SBRT regimen $[12,13,15]$. Timmerman et al. reported that patients with central lesions treated to a total dose of 60-66 Gy in three fractions have an 11-fold increased risk of severe toxicity, compared to patients with peripheral lesions [12]. Song et al. observed a 33\% incidence of grades 3-5 pulmonary toxicities when treating central lesions in 3-4 consecutive days with daily doses of 10-20 Gy [13]. In the study by Fakiris et al., grades 3-5 toxicity occurred in $27 \%$ of patients with central lesions, compared to $10 \%$ of patients with peripheral tumors treated with a dose of 60-66 Gy in three fractions [15]. However, acceptable levels of early pulmonary toxicity have been reported for central lung lesions treated with less aggressive fractionation schemes such as 50-60 Gy in eight to ten fractions [16], 50 Gy in five fractions [17], $48 \mathrm{~Gy}$ in four fractions [18] and $60 \mathrm{~Gy}$ in eight fractions [19]. In all these studies, lung lesions are categorized as central or peripheral based solely on the nearest distance from the main bronchial tree. All lesions located in a zone $<2 \mathrm{~cm}$ away from the bronchial tree are defined as central, with no distinction between lesions fully enclosed in this zone versus lesions lying at the boundary of this zone.

We believe that the shape of the target, its volume, and its geometric extension relative to the bronchial tree may play a significant role in predicting toxicity. A more precise definition of lesion location may enable investigators to

Table 3 Average (standard deviation) value for the dosimetric parameters in each group

\begin{tabular}{|c|c|c|c|c|c|}
\hline Group & Esophagus D1 $1_{\mathrm{cc}}(\%)$ & Cord D1 $1_{\mathrm{cc}}(\%)$ & $\begin{array}{l}\text { Resp. Tract } \\
\mathrm{D} 1_{\mathrm{cc}}(\%)\end{array}$ & $\begin{array}{l}\text { Heart } \\
\mathrm{D} 1_{\mathrm{cc}}(\%)\end{array}$ & $\begin{array}{l}\text { Lung } \\
\text { D10\% (\%) }\end{array}$ \\
\hline Risk Level 1 & 8 & 12 & 27 & 16 & 19 \\
\hline Risk level 2 & $12(11)$ & $16(5)$ & $40(33)$ & 25 (19) & $22(13)$ \\
\hline Risk level 3 & $37(14)$ & $24(12)$ & $73(40)$ & $52(29)$ & $39(20)$ \\
\hline Risk level 4 & $62(29)$ & $29(12)$ & $102(31)$ & $53(33)$ & $53(32)$ \\
\hline$p$ value & 0.009 & 0.046 & 0.039 & 0.172 & 0.158 \\
\hline
\end{tabular}

$D 1_{c c}$ largest dose delivered to $1 \mathrm{cc}$ of a given critical structure, $D 10 \%$ largest dose delivered to $10 \%$ of a given critical structure, Resp. Tract respiratory tract, $p$ value Kruskal-Wallis $p$ value 
distinguish subcategories of central lesions which could be safely and effectively treated with more aggressively hypofractionated SBRT protocols. Our results show that the absolute volume of PTV within a distance of $20 \mathrm{~mm}$ from the airways provides a useful parameter to evaluate the risk of toxicity. We expect that risk-adapted strategies, based on a detailed knowledge of target location, may improve the local control rate for non-peripherally located tumors, while maintaining acceptable levels of toxicity.

In order to develop predictive toxicity models incorporating a consideration of the location of the tumor, an accurate definition of tumor location is needed. The main goal of this paper was to provide a simple and robust method to define target location, based on easily quantifiable parameters. To our knowledge, this is the first paper reporting detailed information on these characteristics for patients with central lung tumors treated with SBRT.

\section{Conclusions}

A new method to characterize target location in lung patients was developed. This method enables determination of the degree of centrality of a lesion, and shows statistical concordance with physicians' decisions on treatment dose and fractionation and with doses received by adjacent dosecritical structures. This method establishes a quantitative basis for the relationship between the spatial location of the lesion and anticipated risks of toxicity. Future studies applying this method to analyze clinical results from large cohorts of lung cancer patients are needed.

Conflict of interest The authors declare that they have no conflict of interest.

\section{References}

1. Nagata Y, Takayama K, Matsuo Y, Norihisa Y, Mizowaki T, Sakamoto T, Sakamoto M, Mitsumori M, Shibuya K, Araki N, Yano S, Hiraoka M (2005) Clinical outcomes of a phase I/II study of 48 Gy of stereotactic body radiotherapy in 4 fractions for primary lung cancer using a stereotactic body frame. Int J Radiat Oncol Biol Phys 63(5):1427-1431

2. Lagerwaard FJ, Haasbeek CJ, Smit EF, Slotman BJ, Senan S (2008) Outcomes of risk-adapted fractionated stereotactic radiotherapy for stage I non-small-cell lung cancer. Int J Radiat Oncol Biol Phys 70(3):685-692

3. Gibbs IC, Loo BW Jr (2010) CyberKnife stereotactic ablative radiotherapy for lung tumors. Technol Cancer Res Treat 9 (6):589-596

4. Timmerman R, Paulus R, Galvin J, Michalski J, Straube W, Bradley J, Fakiris A, Bezjak A, Videtic G, Johnstone D, Fowler J, Gore E,
Choy H (2010) Stereotactic body radiation therapy for inoperable early stage lung cancer. JAMA 303(11):1070-1076

5. Dosoretz DE, Katin MJ, Blitzer PH, Rubenstein JH, Galmarini DH, Garton GR, Salenius SA (1996) Medically inoperable lung carcinoma: the role of radiation therapy. Semin Radiat Oncol 6 (2):98-104

6. Kaskowitz L, Graham MV, Emami B, Halverson KJ, Rush C (1993) Radiation therapy alone for stage I non-small cell lung cancer. Int J Radiat Oncol Biol Phys 27(3):517-523

7. Sibley GS, Jamieson TA, Marks LB, Anscher MS, Prosnitz LR (1998) Radiotherapy alone for medically inoperable stage I nonsmall-cell lung cancer: the Duke experience. Int J Radiat Oncol Biol Phys 40(1):149-154

8. Qiao X, Tullgren O, Lax I, Sirzen F, Lewensohn R (2003) The role of radiotherapy in treatment of stage I non-small cell lung cancer. Lung Cancer 41(1):1-11

9. Ding C, Chang CH, Haslam J, Timmerman R, Solberg T (2010) A dosimetric comparison of stereotactic body radiation therapy techniques for lung cancer: robotic versus conventional linac-based systems. J Appl Clin Med Phys 11(3):3223

10. Timmerman RD, Kavanagh BD, Cho LC, Papiez L, Xing L (2007) Stereotactic body radiation therapy in multiple organ sites. J Clin Oncol 25(8):947-952

11. Lim do H, Yi BY, Mirmiran A, Dhople A, Suntharalingam M, D'Souza WD (2010) Optimal beam arrangement for stereotactic body radiation therapy delivery in lung tumors. Acta Oncol 49 (2):219-224

12. Timmerman R, McGarry R, Yiannoutsos C, Papiez L, Tudor K, DeLuca J, Ewing M, Abdulrahman R, DesRosiers C, Williams M, Fletcher J (2006) Excessive toxicity when treating central tumors in a phase II study of stereotactic body radiation therapy for medically inoperable early-stage lung cancer. J Clin Oncol 24 (30):4833-4839

13. Song SY, Choi W, Shin SS, Lee SW, Ahn SD, Kim JH, Je HU, Park CI, Lee JS, Choi EK (2009) Fractionated stereotactic body radiation therapy for medically inoperable stage I lung cancer adjacent to central large bronchus. Lung Cancer 66(1):89-93

14. De La Fuente HT, Vlachaki MT, Herman TS, Hibbitts K, Stoner JA, Ahmad S (2010) Stereotactic body radiation therapy (SBRT) and respiratory gating in lung cancer: dosimetric and radiobiological considerations. J Appl Clin Med Phys 11(1):3133

15. Fakiris AJ, McGarry RC, Yiannoutsos CT, Papiez L, Williams M, Henderson MA, Timmerman R (2009) Stereotactic body radiation therapy for early-stage non-small-cell lung carcinoma: four-year results of a prospective phase II study. Int J Radiat Oncol Biol Phys 75(3):677-682

16. Taremi M, Hope A, Dahele M, Pearson S, Fung S, Purdie T, Brade A, Cho J, Sun A, Bissonnette JP, Bezjak A (2012) Stereotactic body radiotherapy for medically inoperable lung cancer: prospective, single-center study of 108 consecutive patients. Int J Radiat Oncol Biol Phys 82(2):967-973

17. Olsen JR, Robinson CG, El Naqa I, Creach KM, Drzymala RE, Bloch C, Parikh PJ, Bradley JD (2011) Dose-response for stereotactic body radiotherapy in early-stage non-small-cell lung cancer. Int J Radiat Oncol Biol Phys 81(4):e299-303

18. Stauder MC, Macdonald OK, Olivier KR, Call JA, Lafata K, Mayo CS, Miller RC, Brown PD, Bauer HJ, Garces YI (2011) Early pulmonary toxicity following lung stereotactic body radiation therapy delivered in consecutive daily fractions. Radiother Oncol 99 (2):166-171

19. Haasbeek CJ, Lagerwaard FJ, Slotman BJ, Senan S (2011) Outcomes of stereotactic ablative radiotherapy for centrally located early-stage lung cancer. J Thorac Oncol 6(12):2036-2043 